\title{
Arbor
}

\section{El dilema de un gerente de hospital. Una trayectoria y un equilibrio}

necesarios

\section{Alfredo Macho Fernández}

Arbor CLXX, 670 (Octubre 2001), 231-245 pp.

Introducción: la medicina, el ritmo del cambio, el impacto en la sociedad, los profesionales, el equipo directivo, los representantes de los trabajadores, la divergencia, los poderes públicos, el gerente y la gestión.

La gestión en el final de los años 70 y principio de los 80: el desarrollo tecnológico y la administración de recursos.

La gestión desde principios de los años 80 hasta mediados de los 90: la contención de costes y la gestión profesionalizada.

Los 90 y más: la orientación hacia el paciente y la gestión de la excelencia.

\section{Introducción}

El ejercicio de la Medicina ha estado rodeado de componentes imprevisibles e inciertos, con tal carga de intuición, que más bien cabía considerarlo como arte que como ciencia. Pero nunca ha estado carente de actitudes analíticas, que estudiaban respuestas, contrastaban resultados y comparaban estas variables entre distintos profesionales. $\mathrm{Y}$ han sido estas actitudes comparativas, críticas y valorativas las que han permitido avanzar, pues si bien es cierto que la práxis médica no se ha desprendido totalmente de aquellos componentes de incerti- 
dumbre e intuición, el peso relativo que han ido adquiriendo otros elementos ha propiciado una espectacular modificación tanto conceptual como práctica en el avance y desarrollo de la profesión médica.

En los últimos 25 años, se ha avanzado más en la Medicina y en el cuidado de los pacientes que en los siglos precedentes. Nuestra generación ha tenido el privilegio de vivir este cambio y, sobre todo, debemos resaltar que ha sabido adaptarse a este ininterrumpido caminar, siendo a la vez partícipe y en ocasiones protagonista del mismo.

La Medicina ha pasado de ser una actividad que podía ser desempeñada sin contestación, a estar en el punto de mira de la Sociedad, que nos exige resultados, que quiere implicarse en las decisiones que puedan afectarle y aún más, está cada vez mas interesada y preocupada por analizar y valorar si los recursos que se utilizan y que es consciente que sufraga, logran conseguir los resultados previsibles. Pero a cambio de ello, nunca una profesión ha merecido tanta atención, despertado tanto interés, ni ha gozado de esta consideración social, lo que le ha posibilitado para ejercer una gran influencia en beneficio de la salud de la Humanidad.

Este vertiginoso cambio que estamos señalando ha afectado no sólo al conocimiento del estricto ejercicio de la práctica médica, sino que ha ido mucho más lejos. Análogamente a como ha evolucionado la actividad docente e investigadora en nuestras universidades, el ejercicio de la medicina ha pasado de la responsabilidad individual, sobre criterios implícitos, de aprendizaje por entrenamiento y de desarrollo de habilidades personales, al ejercicio de la profesión médica de forma asociada, en equipo, con criterios explícitos (estándares, severidad de procesos, respuestas a los tratamientos) y compartidos (calidad de vida, concepción del paciente como cliente, etc.), que están dando a las profesiones sanitarias una dimensión y una proyección impensables hace pocas décadas.

Que duda cabe que estos cambios han ido exigiendo el desarrollo de nuevos métodos de gestión y dirección. La conformación de los equipos directivos se va ajustando progresiva, aunque lentamente a criterios más profesionales, donde la formación, la experiencia y la capacidad contrastada, van siendo factores cada vez mas determinantes a la hora de su elección. Pero no debemos olvidar que en esta actividad más que en otras muchas, «se ha hecho camino al andar» en el sentido más estricto de la frase. La situación de partida no podía ser más desfavorable, por la ausencia de experiencia previa y la confusión reinante sobre objetivos, criterios y proyecto.

Este hecho fue simultáneo con el inicio del aperturismo, que posibilitó el demandado, anhelado y necesario desarrollo de la representatividad 
sindical. Sin embargo, aún reconociendo sus innegables aportaciones de diversa índole, debemos reconocer que a menudo se mantienen comportamientos y actitudes trasnochados, centrados en la permanente contestación y alejados de la identificación con los objetivos de la organización, lo que propicia ciertas dificultades de entendimiento, de consenso y de capacitación para abordar proyectos innovadores que faciliten la modernización y la actualización de los centros, sin alterar sustancialmente el clima laboral.

No obstante lo cual, pese a esta aparente divergencia de recorridos entre profesionales, representación sindical y directivos, se han conseguido avances muy significativos y relevantes, a un alto coste eso sí, pero al final positivos; porque lo que hasta el momento parece aceptado por todos, es, que nuestro sistema sanitario es un bien que no debemos dilapidar. Sobre cómo debemos hacerlo subsistir y lograr su mejora continua es donde se centra la discrepancia y el debate.

Corresponde a los poderes públicos garantizar que el sistema sanitario ofrezca una respuesta eficiente a las necesidades de salud de los ciudadanos. La influencia de los medios de comunicación, de la asociaciones científicas y ciudadanas, de las instituciones, de la universidad y de la industria, configuran una amalgama de interrelaciones que confluyen en cada centro sanitario de forma que, ahora más que nunca, la figura del gerente ha de centrarse en asumir las exigencias de la sociedad y generar una cultura de gestión eficiente, en definitiva convertirse en el principal impulsor y armonizador para liderar, explicar y acometer la calidad, punto de encuentro y confluencia entre el gerente y los profesionales clínicos. Para su consecución la gestión ha desarrollado una serie de técnicas y habilidades en los centros asistenciales que le han colocado en una posición relevante. Sin embargo esto le exige, con mayor motivo, hablar cuando es preciso claro y alto, actuar siempre con prudencia y mantenerse en la medida de los posible en un plano discreto.

Ha sido trascendental rodearse de equipos con iniciativa, imaginación y capacidad para gestionar la contradicción. Personas dotadas de la capacidad para avanzar, aún en la discrepancia, haciendo gala permanente de actitudes prudentes, equilibradas y racionales, que no den pie a la inseguridad, que minimicen la incertidumbre y que se convirtieran en impulsores tenaces y firmes de los objetivos y de los criterios de calidad, evidencia y equidad.

Hemos querido señalar algunos hechos y connotaciones que han presidido la evolución de la gestión de nuestros hospitales. Podríamos concluir que la característica común y principal constante, en la gestión 
hospitalaria del último cuarto de siglo, ha sido la continua presión por el cambio. Cambios que en ocasiones se han orientado a objetivos y resultados, mientras que en otras únicamente al proceso o al modelo de gestión.

\section{La gestión de los centros sanitarios ante la presión por el cambio}

Nuestro sistema sanitario, al igual que otros, incluso aquellos con estructuras organizativas y de financiación bien distintas al nuestro, ha estado sometido a continuas modificaciones. Pero la dirección hacia la que iban dirigidos estos cambios, posiblemente bien conocida por los responsables de la política sanitaria en cada momento, no siempre fue tan explícita para quienes trabajábamos como gestores de los centros asistenciales como lo es ahora, desde esta visión retrospectiva que nos permite el paso del tiempo.

Para hacer más comprensible esta exposición marcaremos tres puntos en el tiempo en el que sucesivamente aparecieron ciertas características que se fueron incorporando al quehacer diario de los gestores y configuran actualmente el núcleo de su trabajo.

Los últimos años de la década de los 70 y principio de los 80 , se caracterizaron en nuestro país por la aparición de gran número de profesionales excelentemente formados y de tecnologías y estructuras capaces de dar respuesta a nuevas demandas poblacionales, fruto en gran medida del desarrollo económico y sociocultural incipiente en nuestro país. Por ello calificaremos esta época y el entorno en el que se desarrolla, como el desarrollo tecnológico.

A mediados de la década de los años 80 se empieza a constatar el vertiginoso incremento que se va a producir en el gasto durante los años venideros y la dificultad de mantener desde el punto de vista económico, un sistema sanitario en pleno desarrollo y que terminaba de universalizar sus prestaciones. Aparece así la que llamaremos entorno dirigido a la contención del gasto.

Por último, y posiblemente como reacción a los resultados del entorno que había presidido los años anteriores, desde mediados de los años 90 la política sanitaria parece ir dirigida, sin olvidar la necesidad de mantener los objetivos de las dos épocas anteriores, a la evaluación y adecuación en la distribución y utilización de recursos, hacia la búsqueda de la calidad de la atención, orientando nuestras organizaciones hacia las necesidades tanto de las personas que atendemos como hacia los 
El dilema de un gerente de hospital.....

procesos que padecen, en definitiva hacia los pacientes o personas con una enfermedad. Será la época que denominaremos como la orientación al paciente.

Estos tres periodos que en ocasiones han afectado a los objetivos de resultados de las organizaciones hospitalarias y en otras únicamente al proceso o modelo de gestión, conforman la motivación de los equipos de dirección actuales sobre tres líneas de valores superpuestas.

\section{La gestión en el final de los años 70 y principio de los 80: El desarrollo tecnológico y la administración de recursos}

Por el Decreto Ley 3/ 1978 se constituyó el INSALUD como entidad gestora de la Seguridad Social dependiente en ese momento del Ministerio de Sanidad y Seguridad Social, aunque en 1981 pasó a ser dependiente del Ministerio de Trabajo y Seguridad Social en lo competente a gestión económica y de personal, mientras que al entonces ya Ministerio de Sanidad y Consumo quedo reservada la función de tutelar la actividad asistencial. Esta separación de competencias administrativas es un fiel reflejo del modelo de gestión que en este momento se debía ejercer. Se trataba de dar respuesta a las demandas de atención poblacional cuyas necesidades venían determinadas fundamentalmente por los profesionales sanitarios. Unos profesionales que ya arrastraban una excelente formación pregraduada en nuestro país y que en esos momentos contaron con la oportunidad, para ellos y especialmente para toda la población y sin duda para los gestores, de un sistema de formación posgraduada reglada como fue el sistema MIR que dio lugar a una excelente mejora en la cualificación de nuestros profesionales comparables a la de cualquier otro profesional de los países de nuestro entorno.

Sin embargo, la ordenación de la asistencia hospitalaria y extrahospitalaria continuaba basándose en el decreto de 2766/1967 constituyendo las diferentes dependencias patrimoniales y funcionales de los servicios asistenciales un puzzle difícil de encajar que limitaba de forma importante la continuidad de cuidados a los pacientes. Nuestros hospitales distribuidos según un criterio territorial (Comarcales, regionales, provinciales y Ciudades Sanitarias) eran auténticos organismos con autonomía y descentralización, carentes de cualquier tipo de coordinación interhospitalaria o interniveles. Esta descoordinación estaba incluso presente entre los diferentes servicios de cada uno de los hospitales, dirigidos por un Jefe de Servicio, en muchas de las 


\section{Alfredo Macho Fernández}

ocasiones de gran prestigio profesional en su especialidad clínica y escasa o nula formación en gestión. En la mayoría de estos centros la Dirección del hospital era ejercida por uno de los esos Jefes de Servicio, también de gran prestigio profesional dentro de una estructura organizativa totalmente verticalizada y donde su gestión se limitaba mas bien a la administración de recursos.

Pero expuesta así la situación, podría entenderse como fácil y sencilla la labor de quienes dirigían los hospitales en aquellos momentos. Sin embargo, y sin disponer de hechos fácilmente objetivables, quienes vivíamos la sanidad como clínicos en esos momentos podemos aún recordar cómo influían en la toma de decisiones tanto los cambios políticos, que nuestro país estaba viviendo en esos momentos, como el desarrollo de la reforma del Sistema Sanitario, que concretaba hacia dónde o con qué objetivos se estaba gestando desde la demanda de diferentes colectivos. Esta situación social y cultural del país sin duda tuvo su repercusión en la dirección y gestión de los hospitales e hizo que los entonces gestores tuvieran que actuar en muchas ocasiones como mediadores de conflictos entre los diferentes colectivos e ideologías del momento; profesionales sanitarios, población y organizaciones sindicales que comenzaban a tomar gran protagonismo.

Mientras tanto, como podemos ver en la Tabla 1 el gasto sanitario de nuestra atención especializada crecía de forma tan rápida que, aun sin responsabilidad sobre el gasto, la reforma sanitaria que se produciría en los años siguientes ponía de manifiesto la necesidad de profesionalizar la gestión.

Tras esta breve descripción sobre algunos hechos de interés, por la repercusión de los mismos sobre el tipo de gestión con que contaban nuestros centros, podríamos calificarla como cercana a la administración y a la gestión burocrática.

\section{La gestión desde los primeros años 80 hasta mediados de los 90: La contención del gasto y la gestión "profesionalizada»}

Los gestores de las instituciones sanitarias públicas, se encuentran en medio de un conflicto de intereses entre los enfermos, los profesionales sanitarios, los directivos y los políticos. Diferentes encuestas de opinión realizadas entre profesionales sanitarios muestran un acuerdo de la necesidad de reformar del sistema sanitario superior al 98\% (Martín López) (5). Mostrando una mayoría su deseo de optar por la libertad de elección de médico, la compatibilidad del ejercicio de la medicina pública con el privado y la búsqueda de nuevas fórmulas de pago, 
El dilema de un gerente de hospital.....

Tabla 1

Gastos Totales en Salud (\% sobre PIB)

\begin{tabular}{|l|c|c|c|}
\hline & $\mathbf{1 9 6 0}$ & $\mathbf{1 9 9 2}$ & $\mathbf{1 9 9 9}$ \\
\hline Alemania & 4,8 & 8,7 & 10,4 \\
\hline Australia & 4,9 & 8,8 & \\
\hline Austria & 4,4 & 8,8 & 8,0 \\
\hline Bélgica & 3,4 & 8,2 & 8,4 \\
\hline Canadá & 5,5 & 10,1 & \\
\hline Dinamarca & 3,6 & 6,5 & 5,5 \\
\hline España & $\mathbf{1 , 5}$ & $\mathbf{7 , 0}$ & $\mathbf{7 , 7}$ \\
\hline Estados Unidos & 5,3 & 14,0 & \\
\hline Finlandia & 3,9 & 9,4 & 7,7 \\
\hline Francia & 4,2 & 9,4 & 9,8 \\
\hline Grecia & 2,9 & 5,4 & 7,5 \\
\hline Holanda & 3,5 & 9,2 & 8,8 \\
\hline Islandia & 3,5 & 8,5 & \\
\hline Irlanda & 4,0 & 7,1 & 7,3 \\
\hline Italia & 3,6 & 8,5 & 7,7 \\
\hline Japón & 3,0 & 6,9 & \\
\hline Reino Unido & 4,5 & 7,0 & 5,8 \\
\hline Portugal & 2,1 & 4,1 & 4,5 \\
\hline Suecia & 3,9 & 9,2 & 7,4 \\
\hline Suiza & 4,0 & 9,0 & 10,0 \\
\hline
\end{tabular}

como el pago por acto. Desde los ciudadanos, diferentes encuestas mostraban la diversidad en las preferencias de los diferentes colectivos y los programas sanitarios de los distintos partidos políticos mostraban divergencias en criterios claves para los gestores sanitarios como la privatización de la gestión de los centros públicos. Sin embargo, entre todos estos colectivos y tendencias ideológicas se encontraba una propuesta que podríamos considerar aceptada por todos ellos, que era, la necesidad de hacer una gestión mas profesionalizada de los centros hospitalarios.

La Ley General de Sanidad de 1986 legisló por fin, con muchos debates previos, lo que supondría la reforma sanitaria en España. En lo que se refiere a los centros hospitalarios la Ley regula ya la integración de todos los centros en una única red asistencial y constituye como norma la existencia de un hospital como cabecera de Área de Salud. 
Se abre así la coordinación de la asistencia hospitalaria con la extrahospitalaria, aunque con anterioridad y por Decretos leyes ya estaba regulada la integración de los Facultativos de las llamadas Instituciones Abiertas de la Seguridad Social en estos centros. Pero es esta Ley la que indudablemente dió respuestas a las necesidades demandadas por los distintos agentes sociales y puso ante los gerentes nuevos retos como el tener que dar respuesta a un mayor numero de población con los mismos recursos, a estructurar fórmulas que permitieran la participación ciudadana y de los colectivos de la Atención Primaria.

Durante los años que duró la elaboración de la Ley, precedida por numerosos anteproyectos, los centros hospitalarios renovaron paulatinamente sus equipos directivos apareciendo centros de formación como la Escuela de Gerencia Hospitalaria que inició su andadura por el año 82 y que desde el conocimiento de la teoría trato de dar contenidos directivos a profesionales que ya estábamos inmersos en la realidad de la gestión hospitalaria.

Puede que a partir de estas pinceladas de formación que muchos gestores habíamos recibido y de la necesidad de control del gasto público que crecía de forma desmesurada, al igual que lo hacía la demanda, surgieran muchos de los primeros números y cuadros de mando en la mesa de los gerentes de aquella época. Cuadros de mando con datos cuestionados por los profesionales, fabricados a partir de datos recogidos con sistemas de información totalmente artesanales y que hacían que en muchas ocasiones la toma de decisiones se basará más en la intuición que en la información. De aquí surge otra nueva necesidad, el desarrollo de sistemas de información válidos para la gestión. Conocer al menos el volumen de asistencia que prestaban nuestros centros y unos años más tarde la necesidad de conocer con detalle el gasto real y relacionarlo con la producción fue nuestro nuevo reto.

El tratar de conocer el volumen real de la asistencia y la coordinación de la misma entre servicios (mediante herramientas como la historia clínica única por paciente) fueron algunas de las razones que provocaron la aparición de las admisiones centralizadas de pacientes y de los archivos de historias clínicas únicos. Esto, de alguna forma, se vivió por los clínicos como una usurpación de funciones por parte de las gerencias y hubo que vencer las resistencias al cambio que suponía la aparente pérdida de poder para éstos. Aparece así un conflicto cultural entre médicos y gestores que para algunos se entendió como el fin de la «libertad clínica» (11) y un aparente desplazamiento del poder. Sin embargo, los profesionales de la medicina seguían manteniendo el poder de decisión sobre el gasto y la influencia en los pacientes, 
El dilema de un gerente de hospital.....

más allá de las decisiones clínicas que siempre han gozado del criterio de profesionalidad que requieren.

A pesar de los esfuerzos de los gestores, el gasto sanitario continuaba creciendo y el control del mismo se ejercía a través de sistemas de información sobre indicadores que indicaban muy poco (Decreto sobre Rendimiento Hospitalario de 1978), como eran las estancias medias, los índices de ocupación, los índices de rotación y toda la serie de indicadores que propugnaba el Nuevo Modelo de Gestión Hospitalaria (6) de los años 80, que no permitían relacionar el volumen asistencial con el gasto de cada uno de los centros. Todo ello nos llevó a desarrollar y tener que aprender nuevos métodos en gestión que trataban de medir el producto hospitalario.

En la búsqueda de medidas de actividad y producto hospitalario aparecen los sistemas de Indicadores de producto intermedio: UBA = Unidad Básica Asistencia (Cataluña); UPA = Unidad Ponderada Asistencial (INSALUD); EVA = Escala de Valoración Andaluza (SAS); UCA = Unidad de Coste Asistencial (Osakidetza) los indicadores ajustados por casuística y los sistemas de medidas orientados a los resultados como los Grupos Relacionados con el Diagnóstico, los PMCs, el Disease Staging y otros sistemas de medición del case-mix.

$\mathrm{Si}$ en todas las profesiones la formación continuada ha sido una necesidad, en la del gestor de hospitales, donde se partía de cero y donde los cambios que se pedían de los centros eran tan importantes, esta necesidad, sin disponer de centros de formación e inmersos en la gestión diaria de los centros, se hacia mas acuciante.

Los beneficios que se iban obteniendo desde la gestión profesionalizada no fueron percibidos por los profesionales que cada día se sentían más alejados de quienes, a su juicio, tomaban las decisiones y estos fueron desentendiéndose de la gestión incluso de sus propios servicios y de la gestión de la práctica clínica. La pérdida de poder que habían percibido los responsables de los servicios médicos y su alejamiento de cualquier aspecto relacionado con la gestión conllevó el desprestigio e infravaloración de esta disciplina y de los propios gestores.

Paralelamente a finales de los años 80 surge otra norma reguladora sobre la estructura y funcionamiento de los centros hospitalarios (en INSALUD regulado por el Decreto 521/87) que, sin negar otros beneficios, consigue fragmentar aún más la cultura hospitalaria. La división entre las Direcciones médica y de enfermería repercute también en la coordinación del trabajo asistencial.

Los gestores, que por otro lado continuaban sometidos a los requerimientos de la administración para asegurar las necesidades asis- 
tenciales de la población y la adecuada utilización de presupuestos, no conseguían el objetivo de contención del gasto. Además se habían añadido otros problemas que hacían necesario romper las barreras de comunicación que se habían establecido y nuevos métodos de gestión presupuestaria.

\section{Los 90 y más: La orientación de los centros sanitarios} hacia el paciente y la gestión de la excelencia

A partir de 1991 comenzaron algunos intentos para separar la financiación de la provisión de Servicios. En el caso del INSALUD no transferido se definió un Programa-Contrato marco, entre el Ministerio y el INSALUD y Contratos-Programas específicos entre el INSALUD y las Gerencias de Atención Especializada, dirigidos a potenciar la autogestión de las Instituciones y tratando que los presupuestos hospitalarios estuvieran en función de las actividades a desarrollar por cada centro. Se comienza la facturación a terceros y la competencia entre Áreas, como estrategias de mercado interno dirigidas a configurar a los Hospitales como Empresas de Servicios en la búsqueda de la eficiencia. Con estas estrategias se pretendía, trasladar el riesgo desde el financiador al provisor del servicio. Pero los centros hospitalarios, públicos en su mayor parte y especialmente los no transferidos a las Comunidades Autónomas, formando parte de la Administración, estaban sometidos a la Ley de Contratos de Administraciones Públicas y contaban con un régimen de personal estatutario, muy similar al funcionarial. A pesar de ello, se llevan a cabo intentos dirigidos a una gestión más eficiente, estableciendo concertaciones del sector público con el privado y se regulan nuevas fórmulas de gestión y estrategias de atención diferentes a la hospitalización tradicional.

La Ley 15/1997, de 25 de Abril, sobre nuevas Formas de Gestión del Sistema Nacional de Salud estableció la posibilidad de que la gestión de los centros y servicios sanitarios se pudiera realizar directa o indirectamente a través de cualquier entidad de naturaleza o titularidad pública admitida en Derecho. Amparó la gestión a través de entidades públicas empresariales, consorcios, fundaciones $u$ otros entes dotados de personalidad jurídica propia. Fruto de esta normativa y de la Ley 50/1998, de 30 de Diciembre, de Medidas Fiscales, Administrativas y del Orden Social han aparecido en la sanidad de nuestro país, junto a los tradicionales servicios con financiación de la Seguridad Social, determinadas Sociedades Estatales como entidades públicas o privadas, las Fundaciones Públicas y los Consorcios. 
Pero la dificultad de gestión del Hospital como una empresa, no radicaba sólo en las formas de financiación. Las características propias de su misión asistencial, docente e investigadora hacen que sea considerado como una empresa multiproductos muy especial, donde deben convivir (1) la gestión hotelera, la técnica y la clínica. Aunque las dos primeras podrían beneficiarse del modelo y de las técnicas de la gestión industrial, ambas deben estar integradas y al servicio de la tercera, la gestión clínica (Varela). La falta de esta visión integradora de la asistencia había hecho que, hasta casi mediada la década de los 90, la dirección de los hospitales se hubiera centrado básicamente en temas de la operativa, mediante el uso, y en ocasiones abuso, únicamente de herramientas como la bien conocida (DPO) Dirección Participativa por Objetivos. El cambio supuso incorporar los modelos de Dirección y Planificación Estratégica, un método formal que permite abordar de forma sistemática la potencial repercusión de futuro en todas nuestras decisiones y dirigir el hospital hacia el protagonista final, el paciente.

Pero la aplicación de los principios de gestión clínica a los hospitales no sólo va a requerir cambios en los modelos de contratación y de planificación, sino que hace imprescindible el que estos vayan unidos a cambios en su estructura organizativa.

La clásica estructura organizativa de nuestros hospitales (con la división médica, de enfermería y de gestión) podría, en parte, ser considerada como una forma mixta entre la denominada en el mundo empresarial organización divisional y la denominada funcional. En el organigrama divisional las empresas se estructuran en función de una determinada serie o gama de productos y cada división es prácticamente independiente en sus actividades. Mientras que en el organigrama funcional se agrupan en las diferentes funciones (producción, finanzas, etc.). La gestión clínica, donde la actividad debe ser integradora y dirigida a las necesidades del paciente va a requerir desarrollar organigramas matriciales, y la tradicional organización jerárquica funcional y verticalizada, debe convivir con otras formas orientadas a coordinar actividades y a cumplir objetivos dirigidos al paciente.

En cualquier caso, nuestros hospitales, aunque con la Gestión Clínica, lleguen a integrar todas las actividades y dirigir sus esfuerzos a resolver el problema de salud que ha llevado al paciente al hospital, consiguiendo los mejores resultados tanto desde el punto de vista científico técnico como de costes o eficiencia del sistema, no deben olvidar otros aspectos o atributos de la atención que deben cubrir las necesidades 
del paciente como persona. Es ésta la principal característica de lo que hoy conocemos como el hospital orientado al paciente. El avance que a nivel clínico, tanto en el diagnóstico como en tratamiento de las enfermedades, se ha conseguido, tiene que ir acompañado de la cobertura de nuevas necesidades y valores que derivan del cambio social, cultural y económico que se ha producido en nuestra sociedad. Así, problemas éticos, que en otras circunstancias no lo fueron, como las que provienen del desarrollo científico, deben pesar en las decisiones del gestor a la hora de introducir nuevas tecnologías o al tener que enfrentarse a problemas como el rechazo al tratamiento, el respeto a la intimidad, etc..

Tabla 2

Nuevos retos en gestión hospitalaria

\begin{tabular}{|c|l|}
\hline $\begin{array}{c}\text { La rápida aparición de tecnologías } \\
\text { de información y comunicación. }\end{array}$ & $\begin{array}{l}\text { Historia clínica electrónica } \\
\text { La búsqueda de información médica y las } \\
\text { formas de comunicación en la Red } \\
\text { Oportunidades de inversión en tecnologías } \\
\text { emergentes en sanidad } \\
\text { Servicios domiciliarios y asistencia } \\
\text { remota. Home Care }\end{array}$ \\
\hline $\begin{array}{c}\text { Gestión de la demanda: nuevas } \\
\text { alternativas a la hospitalización } \\
\text { tradicional }\end{array}$ & $\begin{array}{l}\text { Aumento de la actividad ambulatoria } \\
\text { Cirugía Ambulatoria } \\
\text { Hospitalización de día } \\
\text { Hospitalización a domicilio } \\
\text { Hospitalización de noche } \\
\text { Hospitalización de cinco días } \\
\text { Hospitalización de fin de semana }\end{array}$ \\
\hline El marketing hospitalario & $\begin{array}{l}\text { La búsqueda de mercados } \\
\text { Análisis de la demanda } \\
\text { La satisfacción de clientes y proveedores }\end{array}$ \\
\hline Nuevas fórmulas de gestión en la & $\begin{array}{l}\text { Disease management } \\
\text { Coordinación sociosanitaria }\end{array}$ \\
\hline & $\begin{array}{l}\text { Ingeniería genética } \\
\text { Enfermedades emergentes } \\
\text { Medicinas Personalizadas }\end{array}$ \\
\hline
\end{tabular}


En definitiva, los 90 y más, título con el que iniciábamos este apartado, y muy posiblemente los 2000 y más, siguen exigiendo el continuo cambio y aprendizaje al que ya estamos habituados los gerentes y que posiblemente es lo que hace de esta tarea una verdadera profesión. En la Tabla 2, y para no extendernos más, se relacionan algunos de los retos, la mayoría para hoy, de todos los que trabajamos en el medio sanitario, pero en los que el buen hacer de un gestor va a suponer el avance o no de la atención especializada. Y ese buen hacer vendrá determinado por saber o no gestionar nuestras organizaciones hacia la excelencia. Es aquí donde, debemos y podemos, aprender del mejor y en este caso es muy válido y de gran ayuda seguir el modelo de excelencia de European Foundation for Quality Management (EFQM) (9). Este modelo se basa en la premisa de que la satisfacción del cliente, la satisfacción de los empleados, los resultados claves de la empresa y un impacto positivo en la sociedad (criterio deseable en cualquier empresa que busque la supervivencia a medio plazo) se consiguen mediante el liderazgo, el desarrollo de una política y estrategia de la organización, una acertada gestión de personal, el uso eficiente de los recursos y una adecuada definición de los procesos, lo que conduce finalmente a la excelencia de los resultados empresariales. El modelo consta de nueve elementos o criterios ( véase Figura 1 ) que permiten la autoevaluación y el seguimiento de nuestros logros para determinar el progreso de la organización hacia la excelencia.

Figura 1

Modelo EFQM de Excelencia
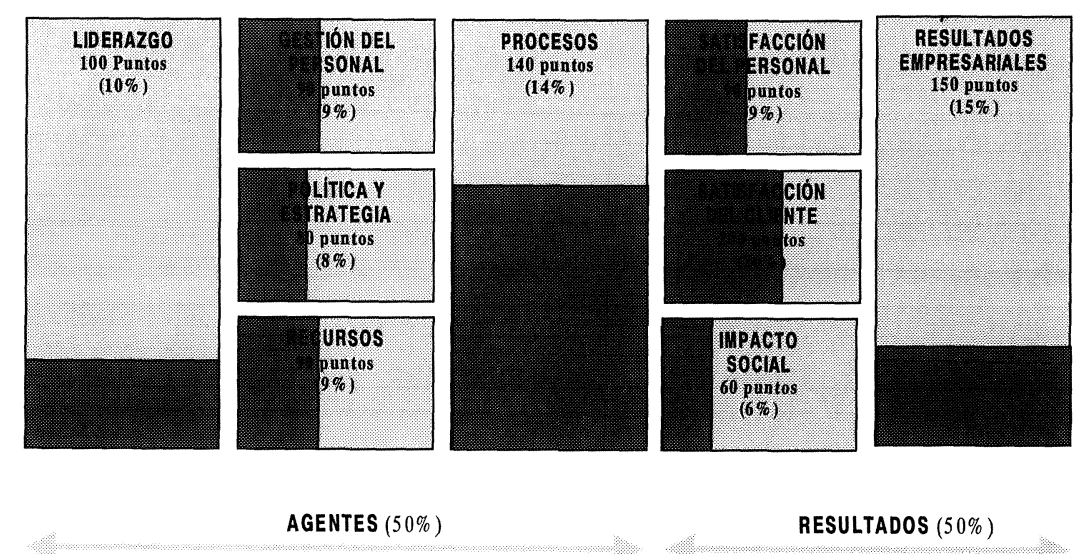

RESULTADOS $(50 \%)$

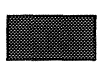

área abordada por la certificación ISO 9000 
Figura 2

La Cultura de la Organización (Modificada de F. Price en Calidad Permanente)

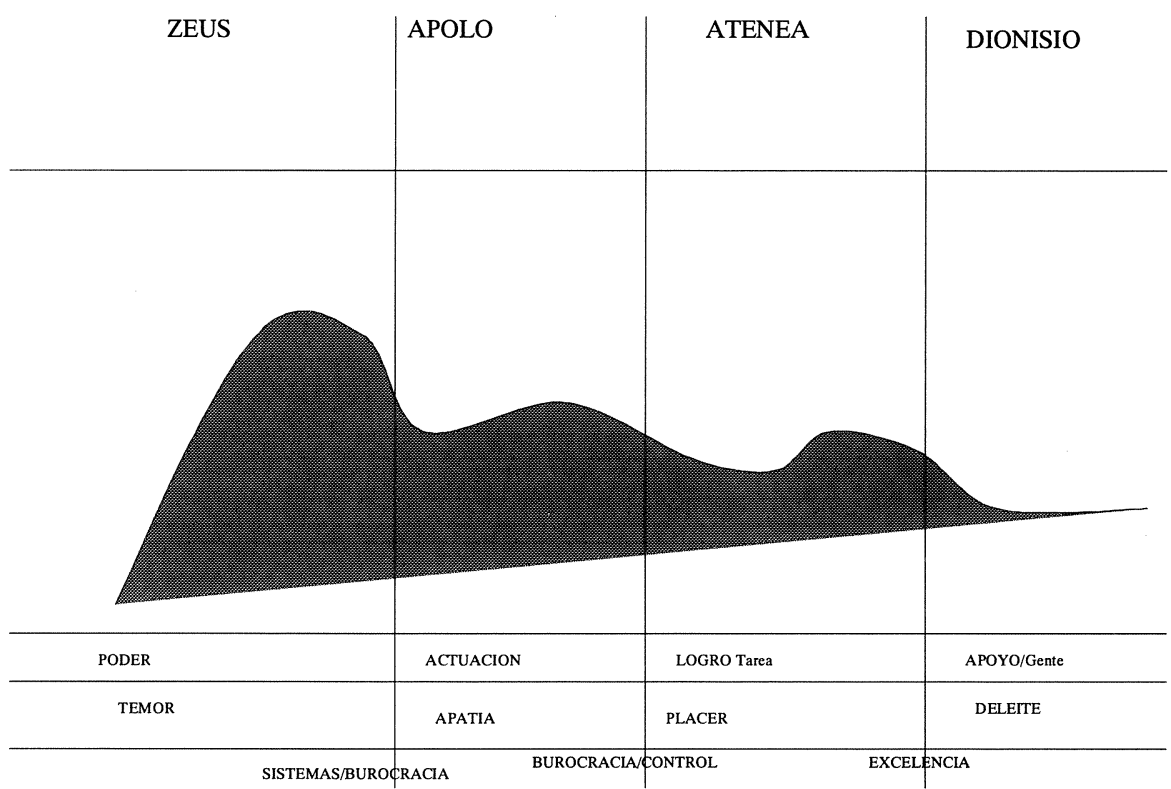

\section{Conclusión}

Por último, como resumen de esta exposición, abordaremos la necesidad de adoptar estilos de dirección acordes con la cultura de cada organización. Expresado de forma más comprensible, nos estamos refiriendo al requisito preliminar de lograr un cambio cultural en las organizaciones para ejercer el estilo de dirección que puede conseguir la excelencia. Para ello, nos remitimos a la Figura 2 (modificada de F.Price) (13) donde a través de lo que llama Modelo Harrison-Handy sobre la cultura de la organización, presidida cada una de ellas por diferentes dioses mitológicos representando los distintos estilos de dirección para cada una de ellas. Así, para las organizaciones con estilos de dirección presididos por el dios Zeus (autocrático y en el mejor de los casos paternalista) sólo funcionarían en culturas donde domina el temor, mientras que aquellas donde predomina la producción de «papel» y la compartimentalización de las funciones, cercanas a las burocracias estarían dirigidas por estilos representados por el dios Apolo, donde 


\section{El dilema de un gerente de hospital.....}

la actuación por si misma, la burocracia, justifica la existencia de las organizaciones. Por otro lado, la diosa Atenea, diosa de la sabiduría y de la guerra, tendría cabida en las «meritrocracias» o el voluntariado, y sólo sería válido en las organizaciones que pretendan su supervivencia a corto plazo. Por último, sólo en las democracias, donde predomina la cultura del apoyo y del trabajo en equipo se conseguiría la excelencia.

\section{Bibliografía}

1 Bohigas, Ll. Control de Gestión en el Hospital. Fundación Avedis Donabedian. Ediciones Decálogo, 1991.

2 Asenjo, M.A., Bohigas, L., Trilla, A. et al. Gestión diaria del hospital. Masson, S.A. Barcelona, 1998.

3 Jiménez, J. Manual de gestión para Jefes de Servicios Clínicos. Conceptos básicos. M.S.D. Madrid, 1997.

4 Elola Somoza, J. Crisis y Reforma de la Asistencia Sanitaria Pública en España. Ed.: Fondo de Investigaciones Sanitarias de la Seguridad Social. Madrid 1991.

5 Del llano. J., Ortún, V., Martín, J.M., Millán, J., Gené, J. Gestión Sanitaria. Innovaciones y desafíos. Masson, S.A. Barcelona, 1998.

6 Subdirección General de Atención Hospitalaria. Nuevo modelo de gestión hospitalaria. Instituto Nacional de la Salud. Ministerio de Sanidad y Consumo. Madrid, 1984

7 Cuervo, J.L., Varela, J., Belenes, R. Gestión de hospitales. Nuevos instrumentos y tendencias. Ediciones Vicens Vivens, S.A. Barcelona, 1994.

8 Temes, J.L., Pastor, V., Díaz, J.L. Manual de Gestión Hospitalaria. Mcgraw - Hill -Interamericana de España. Madrid, 1992.

9 European Foundation for Quality Management. Directrices para el Sector Público. 1997; E.F.Q.M. Bruselas 1996 (Traducido por el Club Gestión de Calidad, Madrid).

10 Juran J.M. Juran y el liderazgo para la Calidad. Un Manual para Directivos. Ed. Díaz de Santos, S.A. Madrid 1990.

11 Williams, A. Economía Sanitaria ¿El fin de la libertad clínica? British Medical Journal. Ed Española : vol IV: Marzo 1989: 66-72.

12 ReLman AS: Assessment and accountability: The third revolution in medical care. N Engl J Med 1988; 319:1220-1220.

13 Price, F. Calidad Permanente: Usando el método Deming. Ed Panorama. Méjico DF 1992. 